\title{
Modelling and control of a water jet cutting probe for flexible surgical robot
}

\author{
Samir Morad Student Member IEEE, Christian Ulbricht, Paul Harkin, Justin Chan, Kim Parker, Ravi \\ Vaidyanathan, Member IEEE
}

\begin{abstract}
-surgical removal of cancerous tissue from the spine is limited by the inability for hand held drills and cutters tool to reach small crevices present in complex bones such as the spinal column. The high speed rotating mechanisms used presently, however, incure stability issues for maneuvering through tortous structures. We report the design and experimental testing of a novel flexible robotic surgical system addressing these issues. The robot consists of a flexible probe, a water jet cutting system, and haptic feedback controller. The water jet cutting system consists of a flexible end effector capable of bending around the spinal column for tissue removal. A new experimental method of controlling the depth of water jet cut is developed. The haptic feedback controller is based on constraint set approach to define 3D boundary constraints based on five key types of constraints. Experimental outcomes of measuring the depth of water jet cut were combined with haptic regional constraints to advance the safety of performing surgical procedures. The reliability, accuracy and performance of the prototype robot were tested in a mock surgical procedure mimicking a surgery on the lower lumbar vertebrae. Results show promise for implementation of water jet cutting for robot surgical spinal procedures.
\end{abstract}

\section{INTRODUCTION}

\section{A. Water jet cutting systems}

High-pressure water jets are classified into two main types: pure water jets (PWJ) and abrasive water jets (AWJ). The basic difference between these two types is the addition of an abrasive medium in AWJ to increase the cutting ability of the water jet. In general, Water-jet technology does not damage the surrounding tissues during the cut because it reaches only a low temperature; it enables high-precision cutting, leaves a clean cut as it instantly flushes out the debris, and decreases bleeding at surgery. However, there is a risk of the jet cutting through the target tissue and then going on to hit the underlying tissues and damaging them.

The initially use of water jet technology was in the mid1880 s with hydraulic mining in which the collection of water from a stream was used to carry the coal out of the working surface of mine [1]. In recent years, the approach of injecting

Supported by UK Engineering and Physical Sciences Research Council

S. Morad, J. Chan and R. Vaidyanathan are with the Department Of Mechanical Engineering, Imperial College, London, phone: (44) 0207594 7020; (samir.morad10; justin.h.chan10; r.vaidyanathan@imperial.ac.uk).

K. Parker is with the Department of Bioengineering, Imperial College, London, south Kensington, (k.parker@imperial.ac.uk).

P. Harkin is with Form Changing Structures, Lincolnshire, UK (paul.harkin@formchangingstructures.com).

C. Ulbricht is with Charing Cross Hospital, London, UK (Christian.Ulbricht@imperial.nhs.uk) abrasives into the flow line between the high pressure pump and the acceleration nozzle $[1,2]$ has rapidly improved the use of water jets and become more common for a growing range of tasks. The water jet technique was adapted in 1982 for medical application by Papachristou and Barters for the resection of the liver [3,4]. Since, this technique was further developed and modified to be used in many medical applications such as neurosurgery, renal surgery, and brain surgery [5-9].

\section{B. Surgical robots and haptic feedback}

The introduction of minimal invasive surgery (MIS) has attributed to a reduction in patient trauma, resulting in faster recovery and lower hospitalisation cost [10]. However, performing MIS, can introduce a range of ergonomic and safety challenges. As technology progresses, the field of medical robotics seems more and more promising with lots of work being done towards implementing robots in the surgical theatre [11]. Robot-assisted surgery promises greater dexterity and higher accuracy by eliminating human error and invoking countless other methods of surgery. However, the absence of feedback eliminates tactile cues, masks force cues and, in some cases, leads to an increase in intra-operative injury [12]. Haptic feedback, which is generally defined as simulated touch interactions between robots, humans, real, remote, or simulated environments, seeks to eliminate this problem.

Active constraint is based on three zone constraints, save, transition, and forbidden zone. This method were first used for a prostatectomy resection and was first clinically applied in 1991 [13]. This 'mechanical' constraint was further developed to provide accuracy and motion constraint for orthopedic knee replacement surgery.

This paper presents a working positional control that incorporates haptic technology structure that adopted for the first time in a flexible robotic device system for spinal surgery. The flexible robot system was developed by the authors[14] to be used in the removal of cancerous tumors surrounding the spinal column. The flexible surgical tool is comprises of two units: a flexible probe and a water jet cutting device. These two integrated units is then mounted on a six degrees of freedom (6DoFs) parallel manipulator, designed by the authors [14], completing the surgical robot system. This system would allow the removal of tumor growth in front of the spinal column without the need for additional invasive entry; thus risk to the patient would be much-reduced.

In this paper, a novel flexible water jet cutter is presented and tested for performance. Water jet cutting tool in surgery; 
have been limited by their capacity to control. In this study a new experimental method of controlling the depth of water jet cut was conducted. In addition, a new method of haptically controlling the depth of water jet cutting is proposed.

\section{SYSTEM DEFENITION}

A 3-3 parallel manipulator platform with $6 \mathrm{DoFs}$ was constructed (Fig.1). The platform is comprised of a fixed top, and a mobile base, connected by six individuallypowered extensible linear actuators.

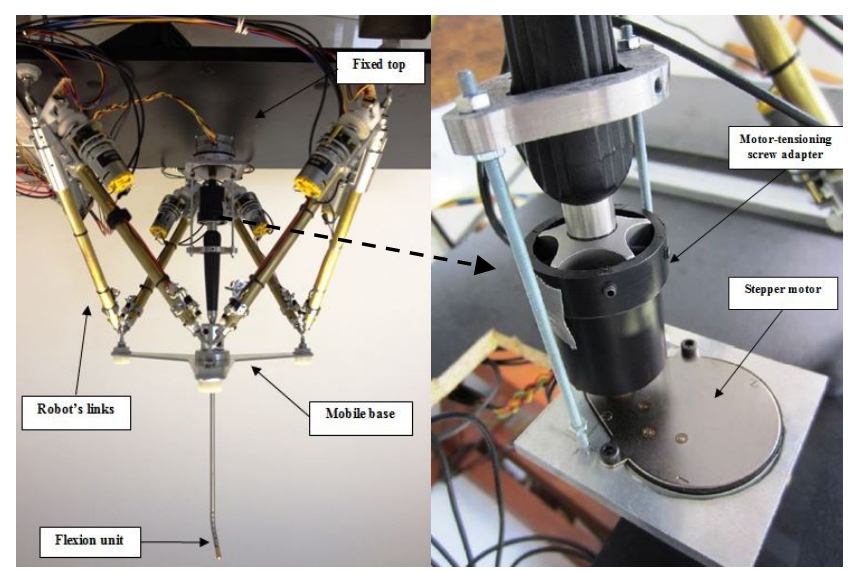

Fig. 1. A 3-3 parallel manipulator with mounted flexible unit at the end effector (left), and the attachment of the stepper motor to the tensioning screw of the flexible tip via an adapter (right).

A flexion unit is a surgical retraction probe with an adjustable articulated distal tip connected to the tensioning screw at the end of proximal handle via cables. The flexible probe was mounted on the platform's end-effector, therefore, this enables the orientation of the probe to be manipulated in the three translational coordinates $(\mathrm{x}, \mathrm{y}$ and $\mathrm{z})$ and three rotational orientations (pitch $\Psi$, roll $\theta$ and yaw $\Phi$ ).

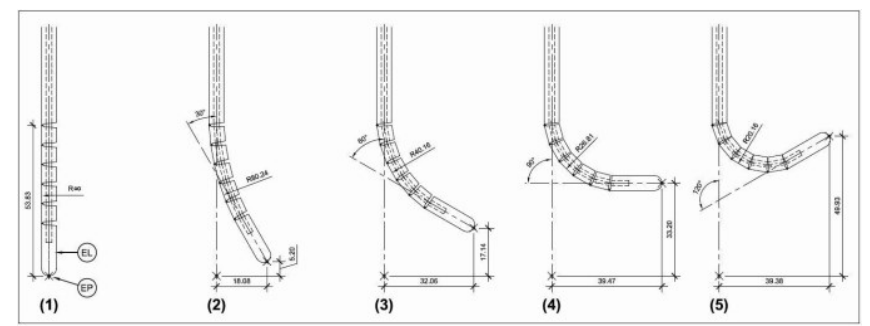

Fig. 2. Stages of bending the flexion distal tip showing the angulation degree for each ascending stage, where EP is the end point of the end link (EL).

When the cables are tightened via tensioning screw, the tip bends to form an approximate arc or J-shape, with the radius of the arc diminishing as the tension increases (Fig.2). As is shown in Fig. 1, when the instrument was mounted on the robot platform, a stepper motor was installed at the proximal end to operate the tensioning screw.

The flexible probe was equipped with a water jet cutting system that uses pressurized water to dissect and resect body tissue (Fig. 3).

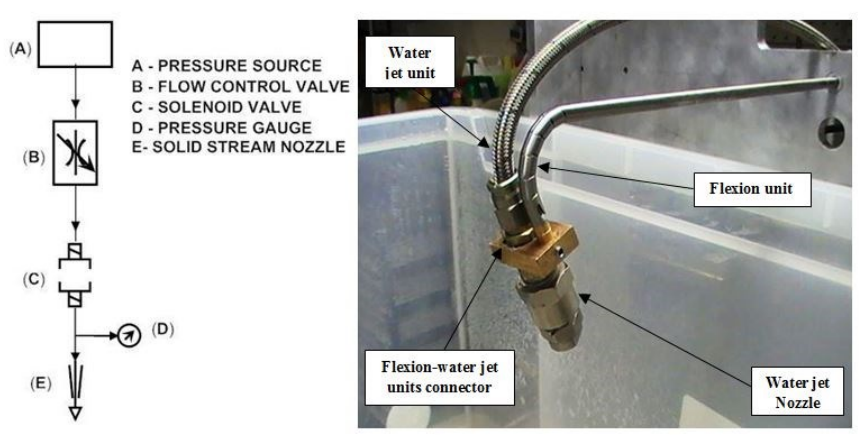

Fig. 3. Schematic of water jet system (left), and the integration of water jet and Flexion units (right).

The setup is connected to a Novint Falcon haptic device controller through a network of electronics, a microcontroller and a computer.

The layout of the electronics used, along with the associated program code that implements full positional control of the platform, can be seen in Fig. 4. The figure illustrates how each electronic component is connected, powered electrically and controlled electronically from the input side through to the output side.

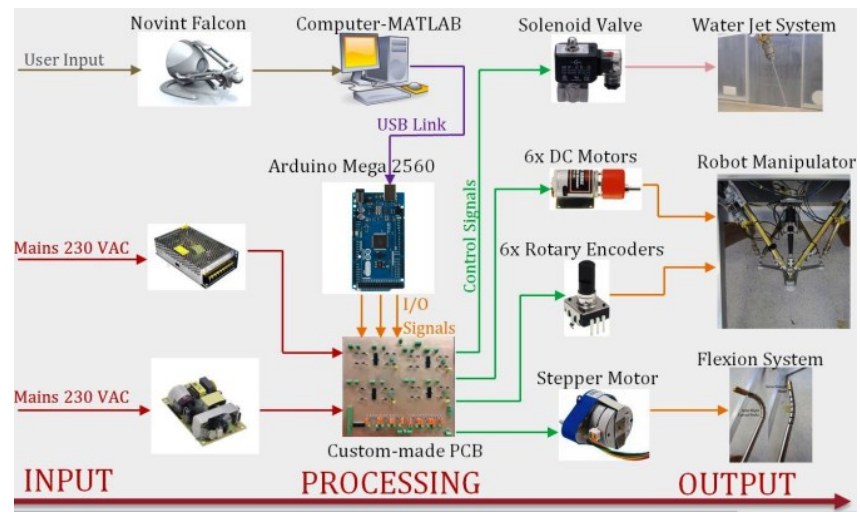

Fig. 4. Component layout of the system

The orientation and position of the flexible probe in $6 \mathrm{DoFs}$ is specified by the user. It is then necessary to calculate the required leg lengths of each of the six platform legs in order for the robot platform to impose the desired orientation of its end-effector. Details of the layout of the electronics used, along with the associated program code that implements full positional control of the platform, are presented in S. Morad et al. [14].

\section{HAPTIC MODELLING}

In this section, two forms of haptic technology were explored, haptic rendering of rigid surfaces and trajectory supervision. It is important to limit the position of the robot to a certain application-defined workspace as unconstrained robot movements can risk dealing severe damage and potentially life-threatening damage to the patient.

\section{A. Haptic Rendering}

Haptic rendering is implemented to restore the surgeon's perception of forces in response to interaction with objects in the surgical environment. Considering the rendering of rigid 
walls; haptic rendering, when viewed from a control theory perspective, is a constrained control problem. The set of constraints and boundaries that are impermissible or inadmissible to the robot are defined in a constraint set. The basic structure of the control loop used for haptic rendering can be seen in Fig. 5 .

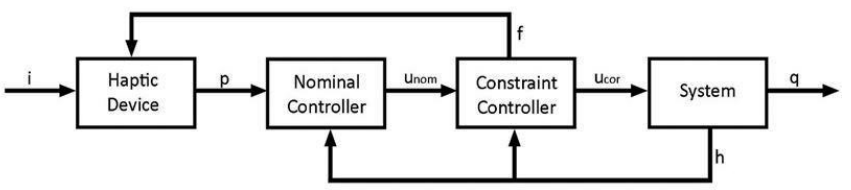

Figure 5: Control loop structure for haptic rendering

A nominal controller, which receives input set $(p)$ from the user interface device, carries out the inverse kinematics and sends the nominal control signals $\left(U_{n o m}\right)$ to the system to achieve the desired position. The constraint controller monitors the state of the system and only becomes active at the boundaries of the constraint set. Here, the constraint controller modifies the nominal control signals $\left(U_{\text {nom }}\right)$ and outputs a corrected control signal $\left(U_{c o r}\right)$ which prevents the system from entering the restricted region. It also computes a force feedback whose magnitude is proportional to the severity of the constraint exceeded. The mathematical equations governing the role of the constraint controller is represents below:

$$
\begin{aligned}
& f_{i}=-\left(i-L B_{i}\right), u_{i c o r}=L B_{i}, \quad \text { for } i<U B_{i} \\
& f_{i}=-\left(i-L B_{i}\right), u_{i c o r}=U B_{i}, \quad \text { for } i>U B_{i} \\
& f_{i}=0, \quad u_{i c o r}=i
\end{aligned}
$$

where UB and LB are upper and lower boundaries respectively, $f$ is force magnitude, and $i$ is $x, y, z$.

Based on the work done by A. Kapoor, et al. [15] in highlighting basic geometric constraints relevant for constrained control in surgical assistant robots, five main types of constraints were defined.

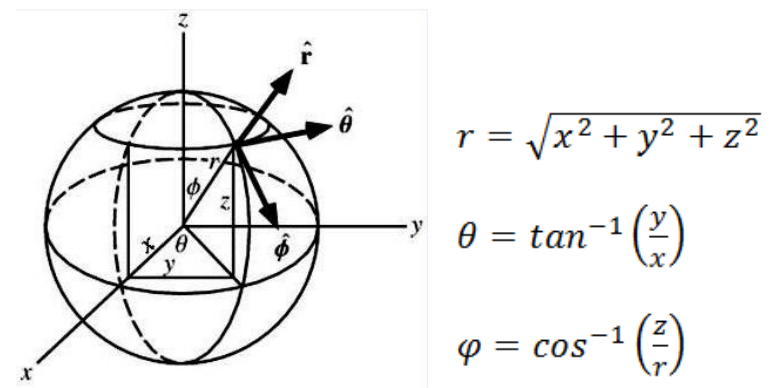

Fig. 6. A sphere with radius $\mathrm{r}$ cantered about the origin $\mathrm{O}$, and the associated equations.

For spherical constraints, as example of one of the geometric constraints, polar coordinates are used to define the system which is natively defined in Cartesian coordinates. Considering a sphere with radius $r$ and cantered about the origin $\mathrm{O}$, as shown in Fig.6. The polar equations for the sphere are numbered 1-3, and an example of constraints set for spherical region is listed in table 1 :

Table 1: Constraint set for a cuboid, spherical and cylindrical constraint

\begin{tabular}{|c|c|l|c|}
\hline Constraint Type & Axis & \multicolumn{1}{|c|}{ Region } & Value \\
\hline \multirow{3}{*}{ Spherical } & \multirow{2}{*}{$\mathrm{x}$} & upper boundary, $U B x$ & $r \cdot \cos \theta \cdot \sin \varphi$ \\
\cline { 3 - 4 } & & lower boundary, $L B x$ & $-r \cdot \cos \theta \cdot \sin \varphi$ \\
\cline { 2 - 4 } & \multirow{2}{*}{$\mathrm{y}$} & upper boundary, $U B y$ & $r \cdot \sin \theta \cdot \sin \varphi$ \\
\cline { 3 - 4 } & & lower boundary, $L B y$ & $-r \cdot \sin \theta \cdot \sin \varphi$ \\
\cline { 2 - 4 } & \multirow{2}{*}{$\mathrm{z}$} & upper boundary, $U B z$ & $r \cdot \cos \varphi$ \\
\cline { 3 - 4 } & & lower boundary, $L B z$ & $-r \cdot \cos \varphi$ \\
\hline
\end{tabular}

\section{B. Trajectory Supervision}

The trajectory supervision of the robot is achieved by integrating a control loop feedback mechanism. In this case a Proportional-Integral-Derivative (PID) controller was implemented to ensure that the robot platform leg lengths extend to the desired length in the ideal time. This is illustrated by the signal $h$ in the Fig. 5. The constraint controller incorporates an additional two constraint sets: the first, a velocity limit set and the second, an allowable threshold set.

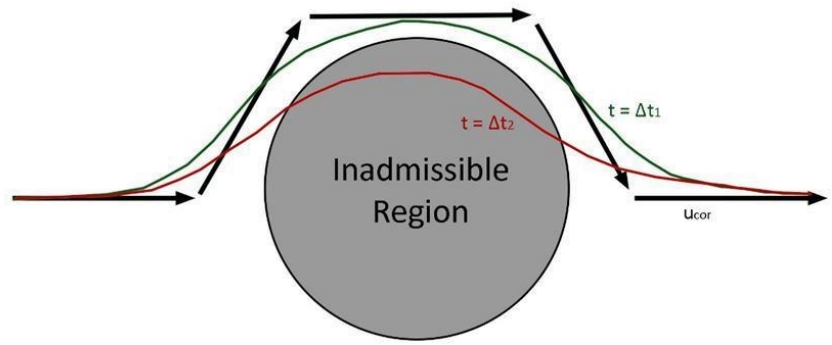

Fig. 7. Illustrative diagram showing the effects of input signal velocity

The system requires time to accelerate and decelerate to reach a target orientation. When the rate of change of the input targets is critically high, the system will not have enough time to respond to the target orientations in an acceptable manner. To illustrate this, Fig.7 shows the same path inputs of $U_{c o r}$ in black arrows. The green trajectory is for an input velocity which is below the critical velocity. The resultant trajectory, shown in red, shows the path of the robot's end-effector crossing the inadmissible region. To avoid this, the input velocity must be limited to be below the critical value at all times. This is done by incorporating a velocity limit constraint that generates a haptic force that slows the user down if the critical velocity is exceeded. The mathematical equations governing the role of velocity limit constraint controller is represents below:

$$
\begin{array}{ll}
f_{i}=-\left(V_{i}+V \max _{i}\right), & \text { for } V_{i}<\operatorname{Vmax}_{i}-f_{t} \\
f_{i}=-\left(V_{i}-V \max _{i}\right), & \text { for } V_{i}>V \max _{i}+f_{t}
\end{array}
$$

Where $f$ is force magnitude, $V$ is velocity, $f_{t}$ is threshold force, and $i$ is $x, y, z$. 


\section{WATER JET SYSTEM: MODELLING AND TESTING}

\section{A. Performance modelling}

The water jet system was set up in the exact configuration as shown in Fig. 3. Quantifiable parameters of the actual system are obtained so that appropriate calculations can be carried out. The values obtained are compared with the predicted values gained from the calculation and flow analysis simulation (Fig. 9). Hence, the flow rate (Q) and pressure of the water jet $(\mathrm{P})$ was measured. The flow rate and pressure were expected to vary based on the discharge flow valve opening. This was done by measuring both variables at every 0.25 revolution of the discharge flow valve knob from fully close to three revolutions. Fig. 8 summarises the results of the test.

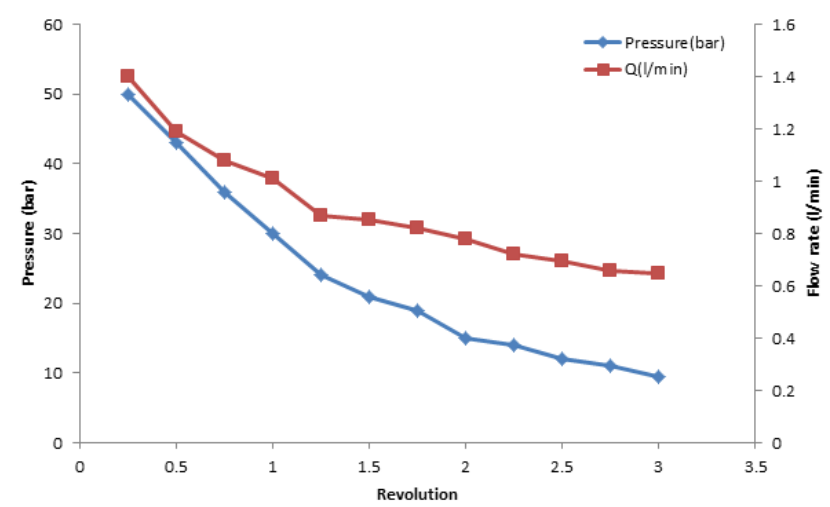

Fig. 8. Plot shows the relationship between flow pressure and flow rate of the water jet unit.
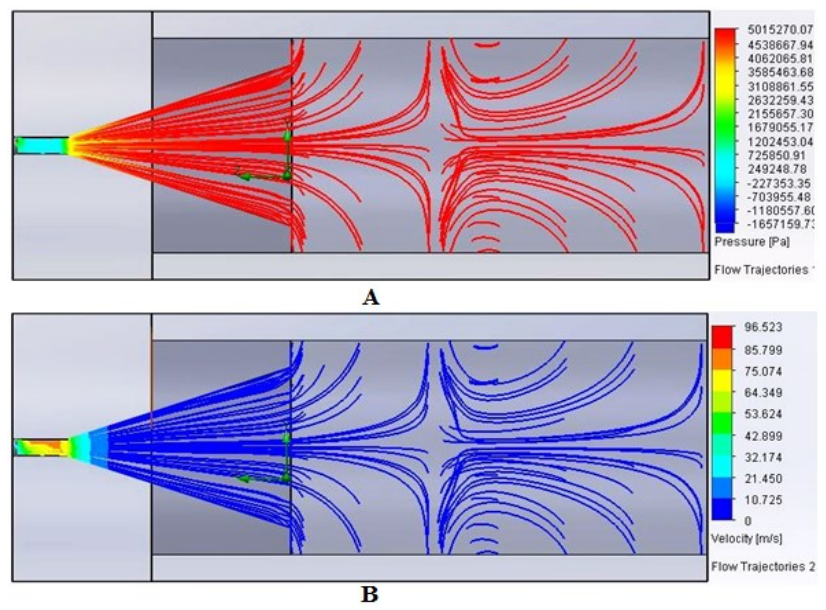

Fig. 9. Flow simulation analysis shows the pressure and velocity distribution for $\varnothing 0.6 \mathrm{~mm}$ diameter nozzle ( $\mathrm{a}, \mathrm{b}$ respectively).

The velocity of the flow is calculated by applying formula (8) using the known outlet pressure, $P$, and density of the fluid, $\rho$ :

$$
v=C_{d} \sqrt{\frac{2 P}{\rho}}
$$

Where $C_{d}$ is the coefficient of discharge, this is the ratio of the actual discharge to the theoretical discharge.

Consequently, we calculate the momentum flow rate $F$ applying:

$$
F\left(k g m / s^{2}\right)=\mu(k g / s) v(m / s)
$$

Where $\mu$ is the mass flow rate.

\section{B. Measure and Control the Depth of Water Jet Cut}

The water jet cutting process result depends on several process parameters. The most important for plain water jet cutting are: water pressure, orifice diameter, nozzle stand-off distance, and the time of cut. These parameters were experimentally measured. The experimental set up is demonstrated in Fig. 10 where a piece of cow topside is used as an object. The depth of cut was measured using a digital caliper with a blunt needle fixed at the tip of the caliper.

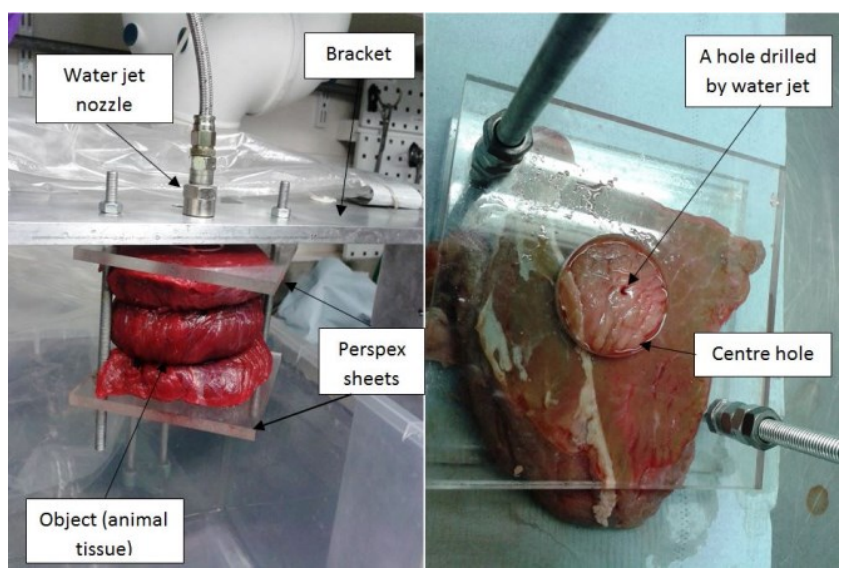

Fig. 10. Experimentally measuring the depth of the water jet cut, experimental setup (left), and a hole drilled by the water jet on an animal tissue (right).

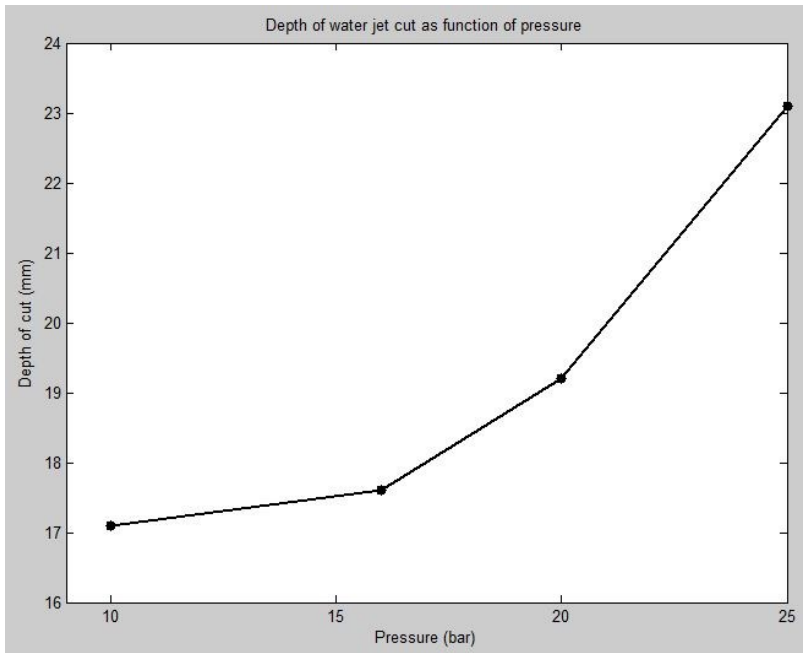

Fig. 11. The depth of the water jet cut as a function of pressure with 3.9 $\mathrm{mm}$ nozzle stand-off distance and $0.84 \mathrm{~mm}$ orifice diameter over $5 \mathrm{~s}$

The first test was conducted to test the effect of the pressure on the depth of cut by varying the source pressure (10-25 bars) over a period of $5 \mathrm{~s}$ keeping the nozzle stand- off distance constant at $3.9 \mathrm{~mm}$ (Fig. 11).

Secondly, the stand- off distance of the water jet nozzle was varied (3.9-23.3 mm) for a selected set of pressures (10-25 bar) that were kept constant over the same period of applied water jet (Fig. 12). 


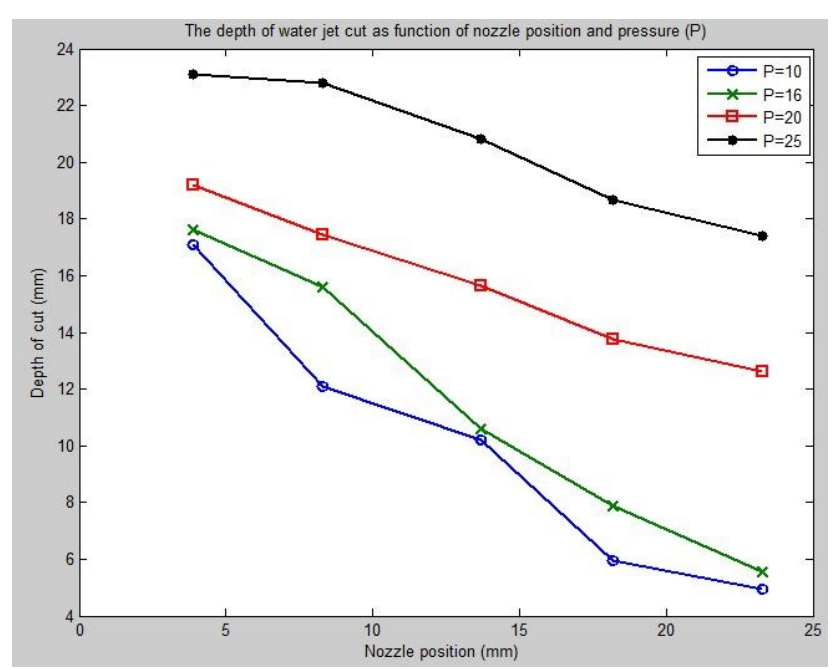

Fig. 12. The depth of the water jet cut as a function of nozzle stand-off distance with $0.84 \mathrm{~mm}$ orifice diameter, applying various set of pressures over $5 \mathrm{~s}$.

The last test was conducted to explore the effect of the nozzle's orifice diameter and time of cut on the depth of water jet (Fig. 13). Two sizes of orifice diameters were used $(0.6 \mathrm{~mm}$ and $0.84 \mathrm{~mm})$; the water jet applied for a period of $5 \mathrm{~s}$ intervals (5- $50 \mathrm{~s})$ with a nozzle stand-off distance of 3.9 $\mathrm{mm}$ and 20 bars of applied $\mathrm{f}$ low pressure.

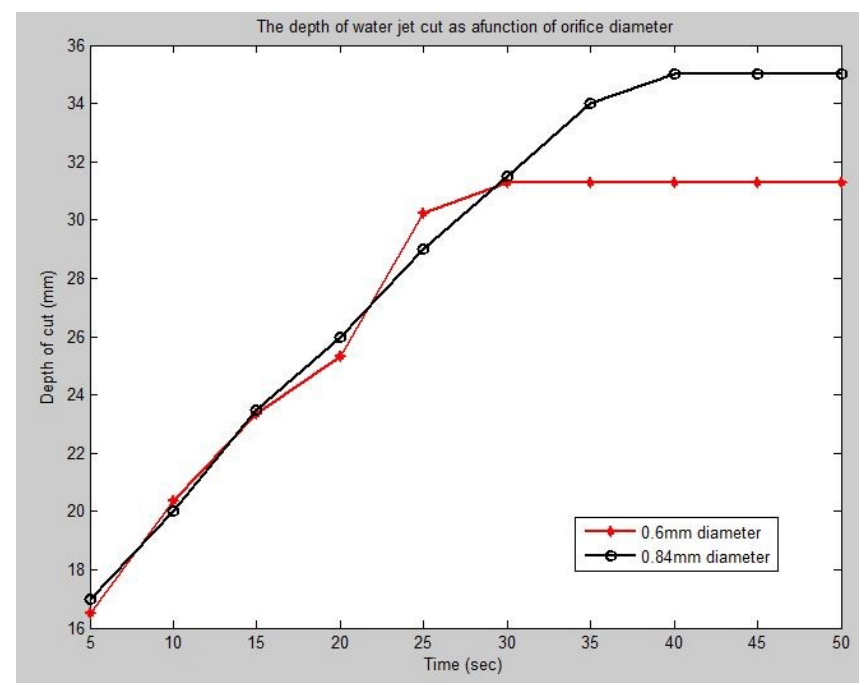

Fig. 13. The depth of water jet cut as a function of nozzle's orifice diameter and the time of applied water jet under flow pressure of 20 bars

\section{Mock Surgery}

A surgical environment was replicated to represent a typical surgical procedure of a surgery on the lower lumbar section of the body (Fig.14). The water jet system was connected to the functional head and the aim of the test was to assess the effectiveness and performance of the robot in the conducting surgical procedure. Overall, the meat was successfully dissected and the robot functioned well in carrying out the procedure. The overall operation time taken was 5 minutes and 20 seconds from start to finish and this time is likely to improve with practice and improvements to the platform hardware.

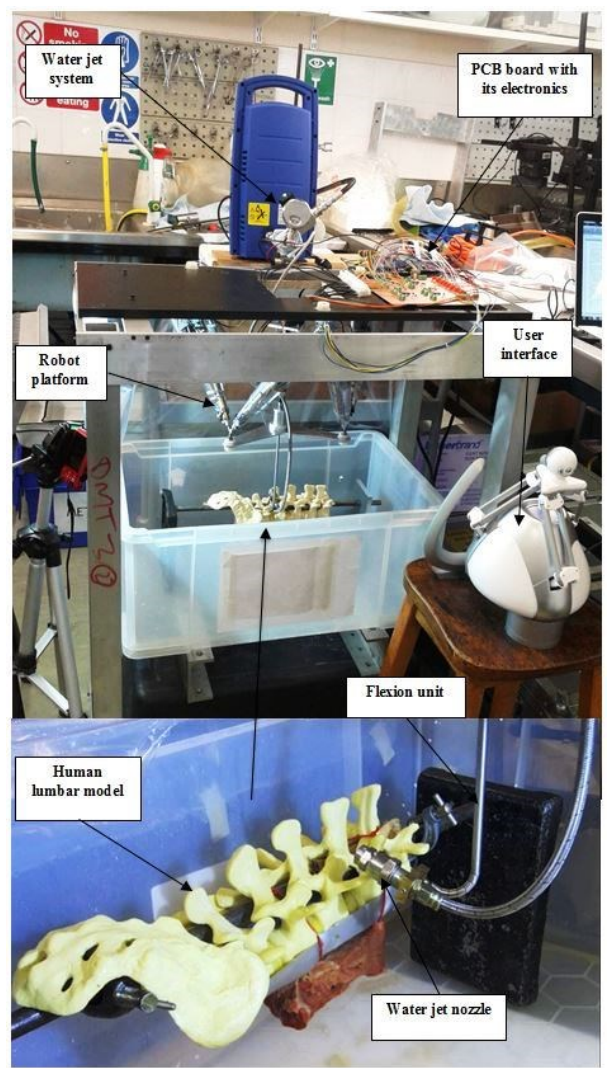

Fig. 14: experimental mock surgery setup with the flexion probe equipped with water jet nozzle.

\section{Haptic Control Of The Depth Of The WATER JET CUT}

The active regional constraint would restrict the movement of the tip from entering a predefined geometric region. This method can be used in the controlling the depth of water jet cut by applying the outcomes obtained from the experimental testing of the water jet cutting animal tissue.

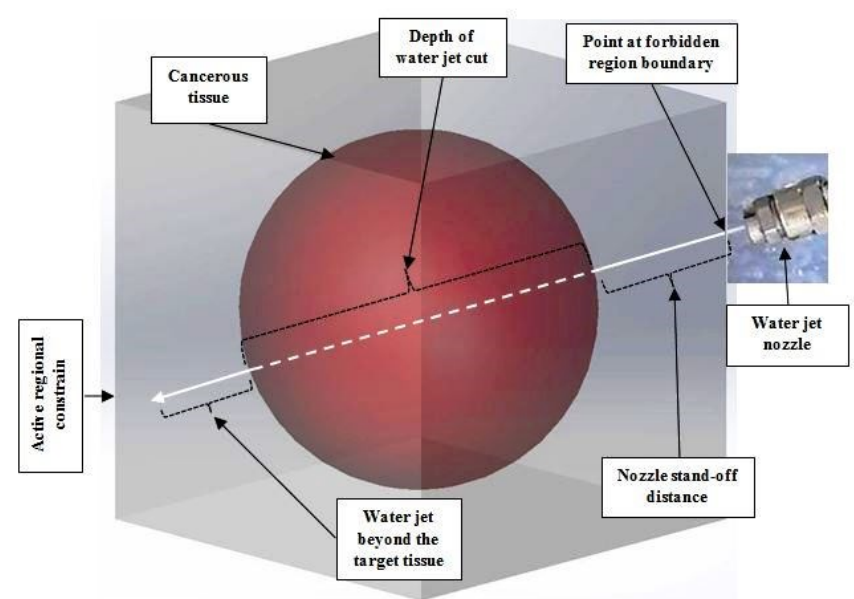

Fig. 15. A plot showing the combination of experimental water jet data and active regional constraint (haptic feedback).

With reference to Fig. 15, assuming that a known size of cancerous tissue (red sphere) is centred inside a predefined cube. The cube represented a regional constraint that 
prevents the tool, in this case the water jet nozzle, from entering the forbidden region. If we position the water jet nozzle on the boundary of the forbidden region, then we know exactly the nozzle stand-off distance, which is the distance between the nozzle and target tissue/ cancerous tumor.

Based on the experimental data analysis and using the relationship between the depth of cut as a function of flow pressure and the stand-off distance of the nozzle (Fig. 13), we can specify the depth of water jet cut as a function of flow pressure and time of the cut. This can be done by using the stand-off distance as a reference and then adjusting the amount of pressure and applied time. Knowing the value of one element of the group of elements that influence the depth of water jet cut, which includes: flow pressure, nozzle diameter, nozzle stand-off distance, and time of cut, would be sufficient to determine the other figures

\section{DISCUSSION}

A significant experimental testing was conducted to measure and control the depth of water jet cutting. In this test, the most important parameters that are involved in the pure water jet cutting process have been tested. The parameters, including water pressure, orifice diameter, standoff distance of the nozzle, and time of cut, were all measured in relationship to each other using two different nozzle sizes.

The experimental result shows that the depth of the water jet cut is significantly dependent on the various parameters. The depth of cut is directly related to the source pressure, time of cut, and nozzle diameter. The depth of cut is inversely related to the stand-off distance. As a result of carrying out the previous experimental tests and outcomes, we are now able to define the depth of water jet cut, or the jet effective length in which it starts to cut the tissue, for certain known parameters this include orifice diameter, stand-off distance, source pressure, and time. The most consistent parameter that affects the depth of water jet cutting depth is the time of applied water. The experimental data (Fig. 13) shows that a constant increase of applied time gives a constant increase in the depth of cut. The data shows that an interval of $5 \mathrm{~s}$ increase in time results in an interval 3 $\mathrm{mm}$ cutting depth. Therefore, controlling the depth of water jet cut can be done by controlling the time. However, the effectiveness of the above parameters is dependent on the type and the mechanical properties of the target tissue such as the tensile strength, compressive strength, modulus of elasticity and hardness. An increase in any of these properties will increase the strength of the material and thus the resistance to a water jet. Therefore, would be necessary to specify the properties of targeted tissue in order to apply the water jet system in surgical environments safely.

\section{CONCLUSION}

We have developed a surgical robotic system that could be used in spinal surgery procedures, with promising early results. Successful feasibility tests, modelling, and simulations were all undertaken. We developed and test a method to determine the depth of water jet cut such that it does not affect (hit) the surrounding tissues. A working positional control structure that incorporates haptic technology in a flexible surgical robotic device was presented.

In this paper we established and proposed the first step in the development of a system that implementing a haptic method of controlling the depth of water jet cut.

The next step is to advance the testing and the modelling of the haptically controlling the depth of water jet cut.

\section{REFERENCES}

[1] D.A. Summers, Waterjetting Technology1995, London: E\&FN Spon.

[2] M. A. Hashish, M.J.K.irby, Y-H. Pao, Method and apparatus for forming a high velocity liquid abrasive jet, in US Patent1987, Flow Industries, Inc.: USA.

[3] K. Schwieger, V.Carrero, R. Rentzsch, A. Becker, N. Bishop, E. Hille, H. Louis, M. Morlock, M. Honl, Abrasive water jet cutting as a new procedure for cutting cancellous bone--in vitro testing in comparison with the oscillating saw. J Biomed Mater Res B Appl Biomater, 2004. 71(2): p. 223-8.

[4] D.N. Papachristou, R.Barters, Resection of the liver with a waterjet. British Journal Of Surgery, 1982. 69: p. 93-94.

[5] B.G. Persson, B.Jeppson, K. Tranberg, K. Roslund, S. Bengmark Transection of the liver with a water jet. Surg Gynecol Obstet, 1989. 168: p. 267-268.

[6] Y. Une, J.Uchino, T. Horie, Y. Sato, K. Ogasawara, A. Kakita, F. Sano Liver resection using a water jet. Cancer Chemother Pharmacol 1989. 23(S74-S77).

[7] H.U. Baer, G.J.Maddren, L.H.Blumgart, New water-jet dissector: initial experience in hepatic surgery. Br. J. Surg, 1991. 78: p. 502503.

[8] J. Piek, J. Ortel, M.R. Gaab, Waterjet dissection in neurosurgical procedures: clinical results in 35 patients. Journal of Neurosurgery, 2002. 96: p. 690-696.

[9] J. Hubert, E. Mourey, J. M. Suty, A. Coissard, J. Floquet, and P. Mangin, Water-jet dissection in renal surgery: experimental study of a new device in the pig. Urol Res, 1996. 24: p. 355-359.

[10] M. Shoham, M.Burman, E. Zehavi, L.Joskowicz, E.Batkilin, and Y. Kunicher, Bone-Mounted Miniature Robot for Surgical Procedures: Concept and Clinical Applications. IEEE Transactions on Robotics and Automation, 2003. 19(5): p. 893 - 901.

[11] A.M. Okamura, Haptic Feedback in Robot-Assisted Minimally Invasive Surgery. Curr Opin Urol, 2009. 19(1): p. 102-107.

[12] H. Xina, J.S.Zeleka, H. Carnahanb, Laparoscopic surgery, perceptual limitations and force: A review, in First Canadian Student Conference on Biomedical Computing2006: Kingston, Ontario, Canada.

[13] P-L. Yen, B.L.Davies., Active constraint control for image-guided robotic surgery. J. Engineering in Medicine, 2010. 224 part H(special issue paper): p. 623-631.

[14] S. Morad, C.Ulbricht, P. Harkin, J.Chan, K. Parker, and R. Vaidyanathan. Flexible Robotic Device for Spinal Surgery. in Proceedings of the 2014 IEEE International Conference on Robotics and Biomimetics. 2014. Bali, Indonesia: IEEE.

[15] A. Kapoor, M.Li, R.H. Taylor. Constrained Control for Surgical Assistant Robots. in Proceedings of the 2006 IEEE International Conference on Robotics and Automation. 2006. Orlando, Florida: IEEE. 